\title{
The massive 3-loop operator matrix elements with two masses and the generalized variable flavor number scheme*
}

\section{J. Ablinger ${ }^{a}$, J. Blümlein ${ }^{b}$, A. De Freitas ${ }^{\dagger b}$, A. Goedicke ${ }^{c} ;$ C. Schneider ${ }^{a}$, K. Schönwald ${ }^{b}$ and F. Wißbrock ${ }^{a, b}$}

${ }^{a}$ Research Institute for Symbolic Computation (RISC), Johannes Kepler University, Altenbergerstraße 69, A-4040, Linz, Austria.

${ }^{b}$ Deutsches Elektronen-Synchrotron, DESY, Platanenallee 6, D-15738 Zeuthen, Germany.

${ }^{c}$ Institut für Theoretische Teilchenphysik, Karlsruhe Institute of Technology (KIT),

76128 Karlsruhe, Germany.

\begin{abstract}
We report on our latest results in the calculation of the two-mass contributions to 3-loop operator matrix elements (OMEs). These OMEs are needed to compute the corresponding contributions to the deep-inealstic scattering structure functions and to generalize the variable flavor number scheme by including both charm and bottom quarks. We present the results for the non-singlet and $A_{g q, Q}$ OMEs, and compare the size of their contribution relative to the single mass case. Results for the gluonic OME $A_{g g, Q}$ are given in the physical case, going beyond those presented in a previous publication where scalar diagrams were computed. We also discuss our recently published two-mass contribution to the pure singlet OME, and present an alternative method of calculating the corresponding diagrams.
\end{abstract}

RADCOR 2017 - 13th International Symposium on Radiative Corrections

(Applications of Quantum Field Theory to Phenomenology),

September 24 - 29, 2017

St. Gilgen, Austria

${ }^{*}$ This work was supported in part by the Austrian Science Fund (FWF) grant SFB F50 (F5009-N15) and the European Commission through contract PITN-GA-2012-316704 (HIGGSTOOLS).

$\dagger$ Speaker.

${ }^{\ddagger}$ A. Hasselhuhn in previous publications. 


\section{Introduction}

Massive operator matrix elements (OMEs) constitute a key ingredient in the calculation of heavy quark corrections to deep-inelastic scattering (DIS) structure functions at large virtualities, as they provide a link between the corresponding massless Wilson coefficients and the massive ones. These OMEs are also needed to obtain the transition relations of the variable flavor number scheme (VFNS). Due to the accuracy of the currently available experimental data, the OMEs need to be calculated at three-loop order. Their knowledge is of importance for the precise measurement of the strong coupling constant $\alpha_{s}\left(M_{Z}^{2}\right)$ [1], of the parton distribution functions [2], and of the heavy quark masses $m_{c}$ and $m_{b}$ [3] from the world deep inelastic data.

In a series of publications, we have computed the single mass contributions at three loops to the OME $A_{g q, Q}$ [4], the $T_{F}^{2}$ terms of the gluonic OME $A_{g g, Q}$ [5], the non-singlet contributions as well as all of the associated Wilson coefficients and structure functions [6-9], the pure singlet result [10], and the diagrams in the case of the ladder and $V$ topologies of the OME $A_{Q g}$ [11]. Furthermore, all diagrams contributing to $A_{Q g}$ which result from master integrals obeying first order factorizing differential equations have been completed [12], as well as all color and $\zeta$-value terms, which can be determined using the method of arbitrarily large moments [13]. The logarithmic contributions to all OMEs were given in [14]. Before a series of moments has been calculated for all massive OMEs in Ref. [15].

At three loops, irreducible Feynman diagrams with two fermion loops of different masses appear for the first time. ${ }^{1}$ The contributions from this type of diagrams cannot be ignored, since the mass of the bottom quark is not considerably larger than the mass of the charm quark, which in particular means that both quarks need to be decoupled simultaneously in the VFNS. The renormalization of the OMEs in the 2-mass case has been performed in Ref. [17]. Here also the VNFS has been generalized to the 2-mass case.

In these proceedings, we report on our recent progress in the calculation of these two-mass three-loop contributions to the OMEs $[17,18]$. In Section 2, we study the simplest of these OMEs, namely, $\tilde{A}_{q q, Q}^{(3), N S}$ and $\tilde{A}_{g q, Q}^{(3)}$ (the tilde on top of the OMEs is used to denote the two-mass contributions). In these cases, the dependence of the OMEs on the Mellin variable $N$ and the masses fully factorizes. In Section 3, we show our recent results on the physical diagrams for $\tilde{A}_{g g, Q}^{(3)}$, going beyond the results presented in [17], where a series of scalar diagrams were computed. In Section 4 we discuss the pure singlet case and show an alternative method of computing the Feynman diagrams and the corresponding Feynman integrals to the one given in Ref. [18] and conclude in Section 5 .

\section{Operator matrix elements with a factorizing $N$ and $\eta$ dependence}

The simplest OMEs containing irreducible two-mass contributions are the three-loop non-singlet OME, $A_{q q, Q}^{(3), N S}$, and the gluonic OME $A_{g q}^{(3)}$. In the case of these two OMEs, the dependence on the ratio of the masses and the Mellin variable $N$ factorizes completely, unlike the case in all other OMEs, where these variables are intertwined in complicated functions, as we will see later.

\footnotetext{
${ }^{1}$ Reducible 2-mass contributions emerge already at NLO, cf. [16].
} 
All of the diagrams appearing in $A_{q q, Q}^{(3), \mathrm{NS}}$ and $A_{g q}^{(3)}$ contain two massive fermion bubbles, one of which may be rendered effectively massless by using a Mellin-Barnes representation [19-23].

$$
\begin{aligned}
\underset{\mu, a}{\infty} \underset{\nu, b}{\infty} & g_{s}^{2} T_{F} \frac{4}{\pi}(4 \pi)^{-\varepsilon / 2}\left(k_{\mu} k_{v}-k^{2} g_{\mu \nu}\right) \\
& \times \int_{-i \infty}^{+i \infty} d \sigma\left(\frac{m^{2}}{\mu^{2}}\right)^{\sigma}\left(-k^{2}\right)^{\varepsilon / 2-\sigma} \frac{\Gamma(\sigma-\varepsilon / 2) \Gamma^{2}(2-\sigma+\varepsilon / 2) \Gamma(-\sigma)}{\Gamma(4-2 \sigma+\varepsilon)} .
\end{aligned}
$$

This yields similar integrals as the ones appearing in the case where there is one massive and one massless fermionic line [24,25]. One may now combine the denominators of the Feynman integrals using Feynman parameters, integrate the momenta and perform the Feynman parameter integrals in terms of Euler Beta-functions. The OMEs will then be given by a linear combination of contour integrals of the form

$$
\begin{aligned}
I \propto \Gamma & {\left[\begin{array}{c}
f_{1}(\varepsilon, N), \ldots, f_{i}(\varepsilon, N) \\
f_{i+1}(\varepsilon, N), \ldots, f_{I}(\varepsilon, N)
\end{array}\right] } \\
& \times \int_{-i \infty}^{+i \infty} d \sigma \eta^{\sigma} \Gamma\left[\begin{array}{c}
\left.g_{1}(\varepsilon)+\sigma, g_{2}(\varepsilon)+\sigma, g_{3}(\varepsilon)+\sigma, g_{4}(\varepsilon)-\sigma, g_{5}(\varepsilon)-\sigma\right], \\
g_{6}(\varepsilon)+\sigma, g_{7}(\varepsilon)-\sigma
\end{array}\right]
\end{aligned}
$$

where the $f_{j}$ and the $g_{j}$ are linear functions, $N$ is the Mellin variable appearing in the operator insertion Feynman rules, and $\eta$ is the ratio of the square of the masses

$$
\eta=\frac{m_{2}^{2}}{m_{1}^{2}}
$$

where we assume $m_{1}>m_{2}$, i.e., $\eta<1$.

After closing the contour in (2.2) and collecting the residues, the integrals end up being expressed as a linear combination of generalized hypergeometric ${ }_{4} F_{3}$-functions [26]

$$
I=\sum_{j} C_{j}(\varepsilon, N){ }_{4} F_{3}\left[\begin{array}{c}
a_{j, 1}(\varepsilon), a_{j, 2}(\varepsilon), a_{j, 3}(\varepsilon), a_{j, 4}(\varepsilon) \\
b_{j, 1}(\varepsilon), b_{j, 2}(\varepsilon), b_{j, 3}(\varepsilon)
\end{array}\right] .
$$

Here and in the following finite and infinite sums occur which have to be summed. In the more involved cases we thoroughly use difference field and ring theory algorithms [27-35] which are encoded in the package Sigma [36,37]. Since in (2.4) the parameters of the hypergeometric functions depend only on the dimensional regularization parameter $\varepsilon$, their corresponding expansion may be performed with the code HypExp 2 [38]. The results of these expansions are then given in terms of the following (poly)logarithmic functions [39-42],

$$
\left\{\ln (\eta), \ln (1-\eta), \ln \left(\frac{1-\sqrt{\eta}}{1+\sqrt{\eta}}\right), \operatorname{Li}_{2}(\sqrt{\eta}), \operatorname{Li}_{2}(\eta), \operatorname{Li}_{3}(\sqrt{\eta}), \operatorname{Li}_{3}(\eta)\right\} .
$$

\subsection{The flavor non-singlet contribution}

The pole structure of the two-mass contribution to the non-singlet OME can be found from the renormalization procedure, which involves mass, coupling constant and operator renormalization, 
as well as collinear factorization. After the subtraction of the single-mass terms, one obtains [17]

$$
\begin{aligned}
\hat{\hat{\tilde{A}}}_{q q, Q}^{(3), \mathrm{NS}}= & -\frac{16}{3 \varepsilon^{3}} \gamma_{q q}^{(0)} \beta_{0, Q}^{2}-\frac{4}{\varepsilon^{2}}\left[\frac{2}{3} \beta_{0, Q} \hat{\gamma}_{q q}^{\mathrm{NS},(1)}+\gamma_{q q}^{(0)} \beta_{0, Q}^{2}\left(L_{1}+L_{2}\right)\right]-\frac{2}{\varepsilon}\left[\beta_{0, Q} \hat{\gamma}_{q q}^{\mathrm{NS},(1)}\left(L_{2}+L_{1}\right)\right. \\
& \left.+\gamma_{q q}^{(0)} \beta_{0, Q}^{2}\left(L_{1}^{2}+L_{2} L_{1}+L_{2}^{2}\right)+4 a_{q q}^{\mathrm{NS},(2)} \beta_{0, Q}-\frac{1}{3} \hat{\tilde{\gamma}}_{q q}^{(2), \mathrm{NS}}\right]+\tilde{a}_{q q, Q}^{(3), \mathrm{NS}}
\end{aligned}
$$

where the $\gamma_{i j}^{(l)}$ are the anomalous dimensions at $l+1$ loops, $\beta_{0, Q}=-\frac{4}{3} T_{F}$, and

$$
L_{1}=\ln \left(\frac{m_{1}^{2}}{\mu^{2}}\right), L_{2}=\ln \left(\frac{m_{2}^{2}}{\mu^{2}}\right)
$$

The renormalized expression in the $\overline{\mathrm{MS}}$-scheme, treating the heavy quarks in the on-shell scheme, is given by

$$
\begin{aligned}
\tilde{A}_{q q, Q}^{(3), \overline{\mathrm{MS}}, \mathrm{NS}}= & \gamma_{q q}^{(0)} \beta_{0, Q}^{2}\left(\frac{2}{3} L_{1}^{3}+\frac{2}{3} L_{2}^{3}+\frac{1}{2} L_{2}^{2} L_{1}+\frac{1}{2} L_{1}^{2} L_{2}\right)+\beta_{0, Q} \hat{\gamma}_{q q}^{\mathrm{NS},(1)}\left(L_{1}^{2}+L_{2}^{2}\right) \\
& +\left\{4 a_{q q}^{\mathrm{NS},(2)} \beta_{0, Q}+\frac{1}{2} \beta_{0, Q}^{2} \gamma_{q q}^{(0)} \zeta_{2}\right\}\left(L_{1}+L_{2}\right)+8 \bar{a}_{q q}^{\mathrm{NS},(2)} \beta_{0, Q}+\tilde{a}_{q q, Q}^{(3), \mathrm{NS}}
\end{aligned}
$$

Both $\hat{\tilde{A}}_{q q, Q}^{(3), \mathrm{NS}}$ and $\tilde{A}_{q q, Q}^{(3), \overline{\mathrm{MS}}, \mathrm{NS}}$ vanish for $N=1$ at all orders in $\varepsilon$ due to fermion number conservation.

In Figure 1, we show a sample of the diagrams contributing to $A_{q q, Q}^{(3), N S}$. The remaining diagrams are related to these by the exchange $m_{1} \leftrightarrow m_{2}$. In the case of the diagrams 2 and 3 in Figure 1, the pre-factors $C_{j}(\varepsilon, N)$ appearing in Eq. (2.4) will contain a sum arising from the vertex operator Feynman rule (see Section 8.1 of Ref. [15]), which can be evaluated in terms of single harmonic sums using the Mathematica packages Sigma [36,37], Harmonicsums [43-45], EvaluateMultisums and SumProduction [46].

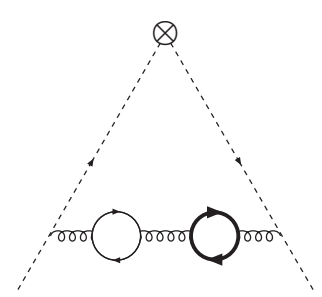

(1)

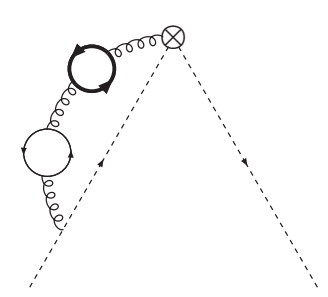

(2)

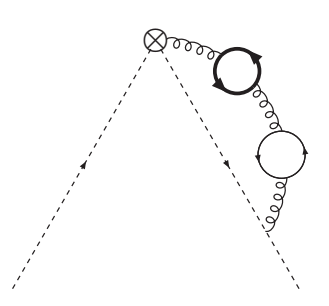

(3)

Figure 1: Diagrams for the two-mass contributions to $\tilde{A}_{q q, Q}^{(3), N S}$. The curly lines denote gluons, the dashed arrow line represents the external massless quarks, while the thick solid arrow line represents a quark of mass $m_{1}$, and the thin arrow line a quark of mass $m_{2}$. We assume $m_{1}>m_{2}$. All diagrams have been drawn using Axodraw [47].

For the constant part, $\tilde{a}_{q q, Q}^{(3), \mathrm{NS}}$, which is the only term in Eq. (2.6) that is not determined by the renormalization prescription, we obtain the following result

$$
\tilde{a}_{q q, Q}^{(3), \mathrm{NS}}=C_{F} T_{F}^{2}\left\{( \frac { 4 } { 9 } S _ { 1 } - \frac { 3 N ^ { 2 } + 3 N + 2 } { 9 N ( N + 1 ) } ) \left[-24\left(L_{1}^{3}+L_{2}^{3}+\left(L_{1} L_{2}+2 \zeta_{2}+5\right)\left(L_{1}+L_{2}\right)\right)\right.\right.
$$




$$
\begin{aligned}
& +\frac{\eta+1}{\eta^{3 / 2}}\left(5 \eta^{2}+22 \eta+5\right)\left(-\frac{1}{4} \ln ^{2}(\eta) \ln \left(\frac{1+\sqrt{\eta}}{1-\sqrt{\eta}}\right)+2 \ln (\eta) \operatorname{Li}_{2}(\sqrt{\eta})-4 \operatorname{Li}_{3}(\sqrt{\eta})\right) \\
& +\frac{(1+\sqrt{\eta})^{2}}{2 \eta^{3 / 2}}\left(-10 \eta^{3 / 2}+5 \eta^{2}+42 \eta-10 \sqrt{\eta}+5\right)\left[\operatorname{Li}_{3}(\eta)-\ln (\eta) \operatorname{Li}_{2}(\eta)\right]+\frac{64}{3} \zeta_{3} \\
& \left.+\frac{8}{3} \ln ^{3}(\eta)-16 \ln ^{2}(\eta) \ln (1-\eta)+10 \frac{\eta^{2}-1}{\eta} \ln (\eta)\right]+\frac{16\left(405 \eta^{2}-3238 \eta+405\right)}{729 \eta} S_{1} \\
& +\frac{4}{3}\left(\frac{3 N^{4}+6 N^{3}+47 N^{2}+20 N-12}{3 N^{2}(N+1)^{2}}-\frac{40}{3} S_{1}+8 S_{2}\right)\left[\frac{4}{3} \zeta_{2}+\left(L_{1}+L_{2}\right)^{2}\right] \\
& +\frac{8}{9}\left(\frac{130 N^{4}+84 N^{3}-62 N^{2}-16 N+24}{3 N^{3}(N+1)^{3}}-\frac{52}{3} S_{1}+\frac{80}{3} S_{2}-16 S_{3}\right)\left(L_{1}+L_{2}\right) \\
& +\left[-\frac{2\left(5 \eta^{2}+2 \eta+5\right)}{9 \eta} S_{1}+\frac{32}{9} S_{2}\right] \ln ^{2}(\eta)-\frac{4 R_{2}}{729 N^{4}(N+1)^{4} \eta} \\
& \left.+\frac{3712}{81} S_{2}-\frac{1280}{81} S_{3}+\frac{256}{27} S_{4}\right\} .
\end{aligned}
$$

Here $S_{\vec{a}} \equiv S_{\vec{a}}(N)$ denote the (nested) harmonic sums [48]

$$
S_{b, \vec{a}}(N)=\sum_{k=1}^{N} \frac{(\operatorname{sign}(b))^{k}}{k^{|b|}} S_{\vec{a}}(k), \quad S_{\emptyset}=1, \quad b, a_{i} \in \mathbb{Z} \backslash\{0\} .
$$

The $R_{i}$ 's are polynomials in $N$ and $\eta$, which we will not show here.

This result is symmetric under the exchange of masses $m_{1} \leftrightarrow m_{2}$, and agrees with the results obtained in $[17]^{2}$ for the individual fixed moments $N=2,4,6$. Eq. (2.9) vanishes for $N=1$ as expected. A similar result is obtained in the case of the transversity contribution, where the change $\gamma_{q q}^{\mathrm{NS}} \rightarrow \gamma_{q q}^{\mathrm{NS} \text {,trans }}[6,51]$ and the corresponding one in the 2-loop OMEs must be performed in Eq. (2.6), cf. [17].

By a Mellin inversion of Eq. (2.9) we obtain the result in $x$-space [17]. In Figure 2, we plot the ratio of the two-mass contribution to the complete $T_{F}^{2}$ term including the single mass results [6] as a function of $x$ and $Q^{2}$, taking the masses of the heavy quarks to be those of the charm and bottom quarks in the on-shell scheme. The two-mass contributions become more important for larger values of $Q^{2}$, where the dependence on the ratio as a function of $x$ becomes almost flat approaching values of $O(0.4)$.

\subsection{The $g q$-contribution}

The genuine two-mass contributions to the $\mathrm{OME} A_{g q, Q}^{(3)}$ can be calculated in a similar way as $A_{q q, Q}^{\mathrm{NS},(3)}$. A sample of the contributing diagrams is shown in Figure 3. There are three more diagrams related to these by the exchange $m_{1} \leftrightarrow m_{2}$.

From the renormalization procedure, one obtains the following pole structure,

$$
\hat{\hat{\tilde{A}}}_{g q, Q}^{(3)}=-\frac{16}{\varepsilon^{3}} \gamma_{g q}^{(0)} \beta_{0, Q}^{2}-\frac{4}{\varepsilon^{2}}\left[3 \gamma_{g q}^{(0)} \beta_{0, Q}^{2}\left(L_{2}+L_{1}\right)+\beta_{0, Q} \hat{\gamma}_{g q}^{(1)}\right]+\frac{1}{\varepsilon}\left[-6 \gamma_{g q}^{(0)} \beta_{0, Q}^{2}\left(L_{2}^{2}+L_{1} L_{2}+L_{1}^{2}\right)\right.
$$

\footnotetext{
${ }^{2}$ See also $[49,50]$.
} 


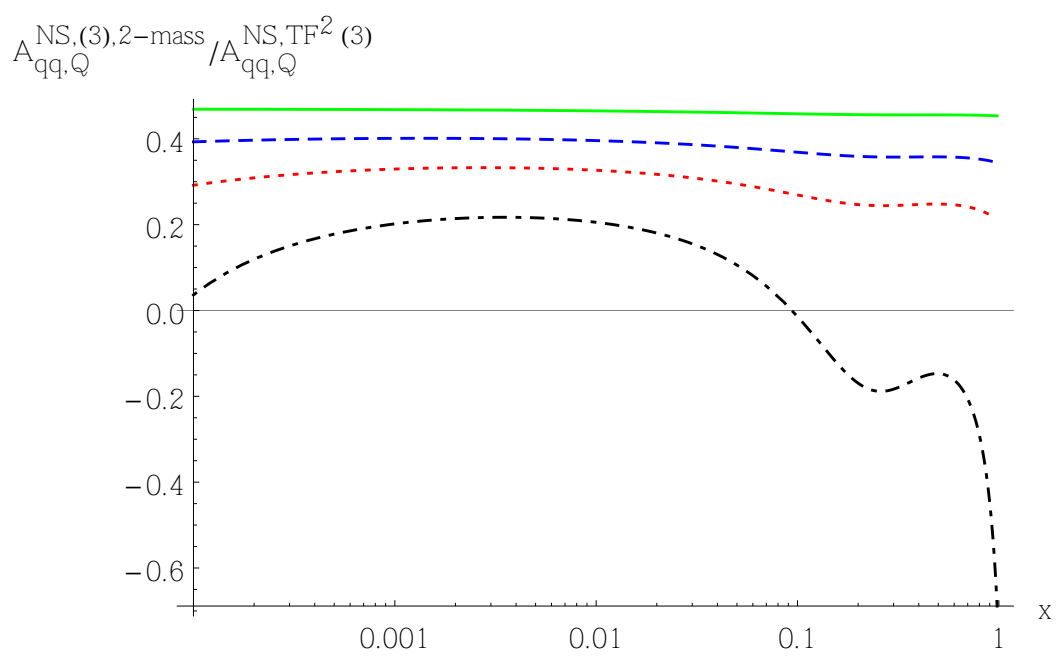

Figure 2: The ratio of the genuine 2-mass contributions to $A_{q q, Q}^{(3), \mathrm{NS}}$ to the complete $T_{F}^{2}$-part of massive 3-loop OME $A_{q q, Q}^{(3), \mathrm{NS}}$ as a function of $x$ and $Q^{2}$, for $m_{c}=1.59 \mathrm{GeV}, m_{b}=4.78 \mathrm{GeV}$ in the on-shell scheme. Dash-dotted line: $\mu^{2}=30 \mathrm{GeV}^{2}$; Dotted line: $\mu^{2}=50 \mathrm{GeV}^{2}$; Dashed line: $\mu^{2}=100 \mathrm{GeV}^{2}$; Full line: $\mu^{2}=1000 \mathrm{GeV}^{2}$. The single mass contributions are given in Ref. [6]; from Ref. [17].

$$
\left.-3 \beta_{0, Q} \hat{\gamma}_{g q}^{(1)}\left(L_{2}+L_{1}\right)+\frac{2}{3} \hat{\gamma}_{g q}^{(2)}-12 a_{g q}^{(2)} \beta_{0, Q}\right]+\tilde{a}_{g q, Q}^{(3)}
$$

and the renormalized operator matrix element in the $\overline{\mathrm{MS}}-$ scheme reads

$$
\begin{aligned}
\tilde{A}_{g q, Q}^{(3), \overline{\mathrm{MS}}}= & \gamma_{g q}^{(0)} \beta_{0, Q}^{2}\left(2 L_{2}^{3}+2 L_{1}^{3}+\frac{3}{2} L_{2}^{2} L_{1}+\frac{3}{2} L_{1}^{2} L_{2}\right)+\frac{3}{2} \beta_{0, Q} \hat{\gamma}_{g q}^{(1)}\left(L_{2}^{2}+L_{1}^{2}\right) \\
& +\left\{6 a_{g q}^{(2)} \beta_{0, Q}+\frac{3}{2} \gamma_{g q}^{(0)} \beta_{0, Q}^{2} \zeta_{2}\right\}\left(L_{2}+L_{1}\right)+12 \bar{a}_{g q}^{(2)} \beta_{0, Q}+\tilde{a}_{g q, Q}^{(3)}
\end{aligned}
$$

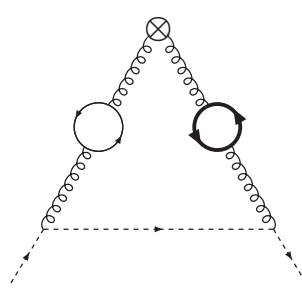

(1)

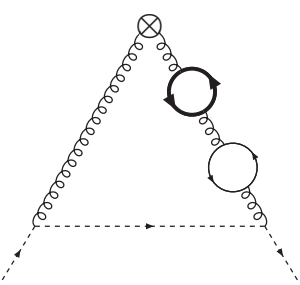

(2)

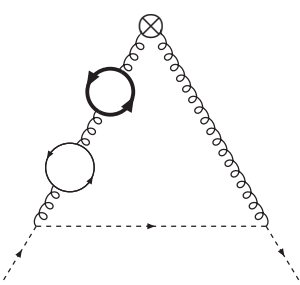

(3)

Figure 3: Diagrams for the two-mass contributions to $\tilde{A}_{g q, Q}^{(3)}$. The curly lines denote gluons, the dashed arrow line represents the external massless quarks, while the thick solid arrow line represents a quark of mass $m_{1}$, and the thin arrow line a quark of mass $m_{2}$. We assume $m_{1}>m_{2}$.

One obtains the following result for the constant part of the two-mass unrenormalized OME,

$$
\tilde{a}_{g q, Q}^{(3)}=C_{F} T_{F}^{2} \frac{1+(-1)^{N}}{2}\left\{p _ { g q } ^ { ( 0 ) } \left[16\left(L_{1}^{3}+L_{2}^{3}+\left(L_{1} L_{2}+2 \zeta_{2}+\frac{26}{3}\right)\left(L_{1}+L_{2}\right)\right)\right.\right.
$$




$$
\begin{aligned}
& -\frac{4}{3 \eta^{3 / 2}}\left((1+\sqrt{\eta})^{2} R_{8} \operatorname{Li}_{3}(-\sqrt{\eta})-(1-\sqrt{\eta})^{2} R_{9} \operatorname{Li}_{3}(\sqrt{\eta})\right)-\frac{16}{9} \ln ^{3}(\eta) \\
& +\left(\frac{2(1+\sqrt{\eta})^{2}}{3 \eta^{3 / 2}} R_{8} \operatorname{Li}_{2}(-\sqrt{\eta})-\frac{2(1-\sqrt{\eta})^{2}}{3 \eta^{3 / 2}} R_{9} \operatorname{Li}_{2}(\sqrt{\eta})-\frac{20}{3 \eta}\left(\eta^{2}-1\right)\right) \ln (\eta) \\
& +\left(\frac{(1+\sqrt{\eta})^{2}}{6 \eta^{3 / 2}} R_{8} \ln (1+\sqrt{\eta})-\frac{(1-\sqrt{\eta})^{2}}{6 \eta^{3 / 2}} R_{9} \ln (1-\sqrt{\eta})-\frac{16}{3} S_{1}\right) \ln ^{2}(\eta) \\
& \left.-\frac{64}{27} S_{1}^{3}-\frac{128}{27} S_{3}-\frac{64}{3}\left(\zeta_{2}+\frac{1}{3} S_{2}\right) S_{1}-\frac{128}{9} \zeta_{3}\right]-\frac{R_{10} \ln ^{2}(\eta)}{3 \eta(N-1) N(N+1)^{2}} \\
& +16\left[-\frac{1}{(N+1)^{2}}+p_{g q}^{(0)}\left(\frac{8}{3}-S_{1}\right)\right]\left(\left(L_{1}+L_{2}\right)^{2}-\frac{4}{3}\left(L_{1}+L_{2}\right) S_{1}\right) \\
& +\left[\frac{32}{3} p_{g q}^{(0)}\left(S_{2}-S_{1}^{2}\right)-\frac{64(8 N+5)}{9(N+1)^{3}}\right]\left(L_{1}+L_{2}\right)-\frac{64 R_{11} S_{1}}{27(N-1) N(N+1)^{3}} \\
& \left.+\frac{64\left(8 N^{3}+13 N^{2}+27 N+16\right)}{27(N-1) N(N+1)^{2}}\left(S_{1}^{2}+S_{2}+3 \zeta_{2}\right)-\frac{8 R_{12}}{243 \eta(N-1) N(N+1)^{4}}\right\}
\end{aligned}
$$

where $p_{g q}^{(0)}$ is the color-stripped 1-loop anomalous dimension,

$$
p_{g q}^{(0)}=\frac{2+N+N^{2}}{(N-1) N(N+1)} .
$$

Again, we will omit the explicit expressions of the polynomials $R_{i}$.

The corresponding $x$-space expression is given in the appendix of Ref. [17]. Again, Eq. (2.13) agrees with previously calculated fixed moments for $N=2,4,6$. The behaviour of the ratio of the genuine 2-mass contribution to the complete $T_{F}^{2}$ 3-loop result for $A_{g q, Q}^{(3)}$ at large values of $Q^{2}$ is similar to the one exhibited in the non-singlet case in Figure 2.

\section{The gluonic operator matrix element $\tilde{A}_{g g}^{(3)}$}

The three-loop contributions to $\tilde{A}_{g g}$ have no effect on the DIS structure functions at NNLO, since only the corresponding two-loop contributions appear in the expressions for the massive Wilson coefficients (see Eq.s (2.20)-(2.24) of Ref. [17]). They are, however, needed in order to obtain the gluonic transition relation of the VFNS at three loops. The calculation of $\tilde{A}_{g g}^{(3)}$ is considerably more elaborate than those of the previous OMEs, and leads to new types of functions, namely, generalized sums in $N$-space and the generalized harmonic polylogarithms with square root letters in the alphabet in $x$-space.

There is a total of 76 irreducible diagrams contributing to the two-mass part of $A_{g g}^{(3)}$, including six diagrams with a ghost in the external lines. Only twelve of them, shown in Figure 4, are truly independent. All other diagrams are related to these either by symmetry, the exchange of masses or reversal of a fermion line.

In Ref. [17], we presented the results for the scalar versions of the first eight diagrams of Figure 4. We have now completed the calculations of all but two of the diagrams in the physical case. The details of how this calculation has been performed will be explained in a future publication. As a preview, we show here the result for one of the diagrams, namely diagram 6 of Figure 4. We get, 


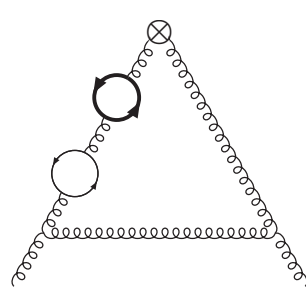

(1)

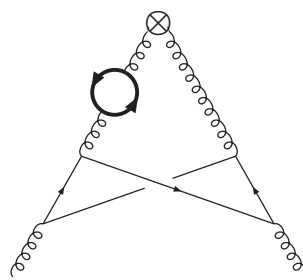

(5)

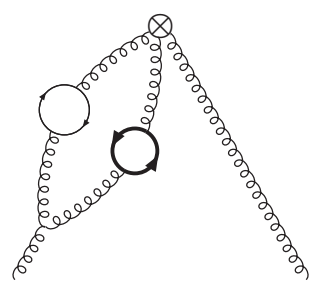

(9)

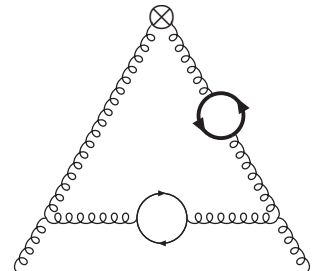

(2)

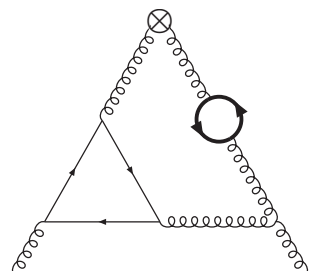

(6)

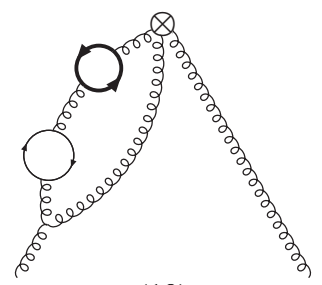

(10)

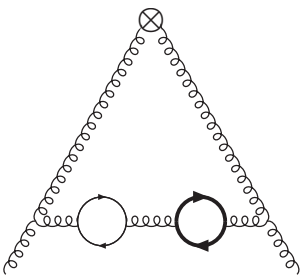

(3)

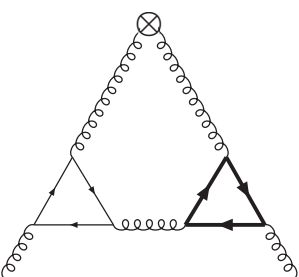

(7)

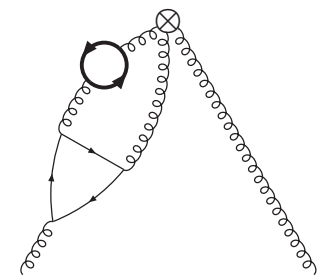

(11)

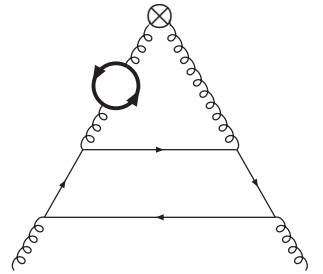

(4)

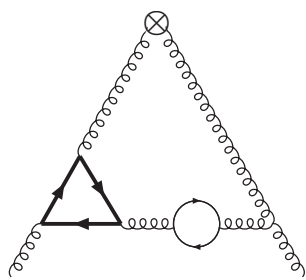

(8)

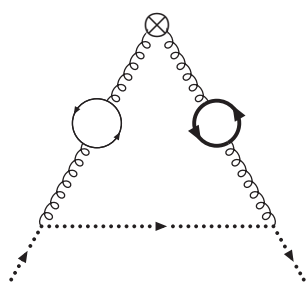

(12)

Figure 4: Independent diagrams for the two-mass contributions to $\tilde{A}_{g g}^{(3)}$. The curly lines denote gluons, the dotted line represents a ghost, the thick solid arrow line represents a quark of mass $m_{1}$, and the thin arrow line a quark of mass $m_{2}$. We assume $m_{1}>m_{2}$.

$$
\begin{aligned}
D_{6}^{B}(N)= & C_{A} T_{F}^{2} \frac{1+(-1)^{N}}{2}\left\{\frac{16\left(29 N^{3}+41 N^{2}+47 N+47\right)}{27(N-1) N(N+1)^{2} \varepsilon^{3}}+\frac{1}{\varepsilon^{2}}\left[\frac{8 P_{5}}{81(N-1)^{2} N^{2}(N+1)^{3}}\right.\right. \\
& \left.-\frac{8\left(13 N^{3}+21 N^{2}+23 N+27\right)}{9(N-1) N(N+1)^{2}} H_{0}(\eta)-\frac{8\left(23 N^{3}+19 N^{2}+21 N+13\right)}{27(N-1) N(N+1)^{2}} S_{1}\right] \\
& +\frac{1}{\varepsilon}\left[\frac{4 P_{6}}{81(N-1)^{3} N^{3}(N+1)^{4}}+\frac{2\left(17 N^{3}-3 N^{2}-5 N-21\right)}{27(N-1) N(N+1)^{2}} S_{2}\right. \\
& +\frac{2\left(23 N^{3}+43 N^{2}+45 N+61\right)}{9(N-1) N(N+1)^{2}} H_{0}^{2}(\eta)+\frac{2\left(29 N^{3}+41 N^{2}+47 N+47\right)}{27(N-1) N(N+1)^{2}} S_{1}^{2} \\
& -\frac{4 P_{3}}{27(N-1)^{2} N^{2}(N+1)^{3}} H_{0}(\eta)+\frac{2\left(29 N^{3}+41 N^{2}+47 N+47\right)}{9(N-1) N(N+1)^{2}} \zeta_{2} \\
& \left.+\left(-\frac{4 P_{4}}{81(N-1)^{2} N^{2}(N+1)^{3}}+\frac{4\left(7 N^{3}-N^{2}-3 N-7\right)}{9(N-1) N(N+1)^{2}} H_{0}(\eta)\right) S_{1}\right] \\
& -\frac{53 N^{3}+109 N^{2}+111 N+163}{27(N-1) N(N+1)^{2}} H_{0}^{3}(\eta)-\frac{23 N^{3}+19 N^{2}+21 N+13}{81(N-1) N(N+1)^{2}} S_{1}^{3}
\end{aligned}
$$




$$
\begin{aligned}
& +\frac{5 N^{3}+11 N^{2}+11 N+17}{9(N-1) N(N+1)^{2}}\left(4 H_{0}(\eta) H_{0,1}(\eta)-4 H_{0,0,1}(\eta)-2 H_{0}^{2}(\eta) H_{1}(\eta)\right) \\
& +\frac{4 P_{8}}{45 \eta(N-1) N(N+1)^{2}}\left\{H_{0}(\eta) S_{1,1}\left(\frac{\eta}{\eta-1}, \frac{\eta-1}{\eta}, N\right)-S_{1,2}\left(\frac{\eta-1}{\eta}, \frac{\eta}{\eta-1}, N\right)\right. \\
& \left.+S_{1,2}\left(\frac{\eta}{\eta-1}, \frac{\eta-1}{\eta}, N\right)+S_{1,1,1}\left(\frac{\eta-1}{\eta}, 1, \frac{\eta}{\eta-1}, N\right)+S_{1,1,1}\left(\frac{\eta-1}{\eta}, \frac{\eta}{\eta-1}, 1, N\right)\right\} \\
& -\frac{2 P_{9}}{405(N-1) N(N+1)^{2} \eta} S_{3}+\frac{P_{14}}{3645(N-1)^{4} N^{4}(N+1)^{5}(2 N-5)(2 N-3)(2 N-1) \eta} \\
& +\frac{P_{15}}{90(N-1) N(N+1) \eta^{3 / 2}}\left(H_{0}(\eta) H_{0,-1}(\sqrt{\eta})+H_{0}(\eta) H_{0,1}(\sqrt{\eta})-\frac{1}{4} H_{0}(\eta)^{2} H_{1}(\sqrt{\eta})\right. \\
& \left.-2 H_{0,0,-1}(\sqrt{\eta})-2 H_{0,0,1}(\sqrt{\eta})\right)+\frac{P_{5}}{27(N-1)^{2} N^{2}(N+1)^{3}} \zeta_{2} \\
& +\frac{2^{-2 N-3}\left(\begin{array}{c}
2 N \\
N
\end{array}\right) P_{16}}{135(N-1) N(N+1)^{2}(2 N-5)(2 N-3)(2 N-1) \eta^{2}}\left[\sum_{i=1}^{N} \frac{2^{2 i}}{\left(\begin{array}{c}
2 i \\
i
\end{array}\right) i^{3}}-\sum_{i=1}^{N} \frac{2^{2 i} S_{1}(i)}{\left(\begin{array}{c}
2 i \\
i
\end{array}\right) i^{2}}\right. \\
& +H_{0}(\eta) \sum_{i=1}^{N} \frac{2^{2 i}}{\left(\begin{array}{c}
2 i \\
i
\end{array}\right) i^{2}}+\sum_{i=1}^{N} \frac{2^{2 i}(\eta-1)^{-i} \eta^{i}}{\left(\begin{array}{c}
2 i \\
i
\end{array}\right) i} S_{1,1}\left(\frac{\eta-1}{\eta}, 1, i\right) \\
& +\frac{1}{2} H_{0}^{2}(\eta) \sum_{i=1}^{N} \frac{2^{2 i}(\eta-1)^{-i} \eta^{i}}{\left(\begin{array}{c}
2 i \\
i
\end{array}\right) i}-H_{0}(\eta) \sum_{i=1}^{N} \frac{2^{2 i}(\eta-1)^{-i} \eta^{i}}{\left(\begin{array}{c}
2 i \\
i
\end{array}\right) i} S_{1}\left(\frac{\eta-1}{\eta}, i\right) \\
& \left.-\sum_{i=1}^{N} \frac{2^{2 i}(\eta-1)^{-i} \eta^{i}}{\left(\begin{array}{c}
2 i \\
i
\end{array}\right) i} S_{2}\left(\frac{\eta-1}{\eta}, i\right)\right]-\frac{2\left(11 N^{3}+47 N^{2}+41 N+89\right)}{27(N-1) N(N+1)^{2}} \zeta_{3} \\
& +\left(\frac{\eta}{\eta-1}\right)^{N} \frac{P_{11}}{540(N-1)^{2} N^{2}(N+1)^{2}(2 N-5)(2 N-3)(2 N-1) \eta}\left[S_{2}\left(\frac{\eta-1}{\eta}, N\right)\right. \\
& \left.+H_{0}(\eta) S_{1}\left(\frac{\eta-1}{\eta}, N\right)-\frac{1}{2} H_{0}^{2}(\eta)-S_{1,1}\left(\frac{\eta-1}{\eta}, 1, N\right)\right] \\
& +\frac{2^{-2 N-4}\left(\begin{array}{c}
2 N \\
N
\end{array}\right) P_{16}}{135(N-1) N(N+1)^{2}(2 N-5)(2 N-3)(2 N-1) \eta^{3 / 2}}\left[8 H_{0,0,-1}(\sqrt{\eta})\right. \\
& \left.+\left(H_{-1}(\sqrt{\eta})+H_{1}(\sqrt{\eta})\right) H_{0}^{2}(\eta)+8 H_{0,0,1}(\sqrt{\eta})-4 H_{0}(\eta)\left(H_{0,-1}(\sqrt{\eta})+H_{0,1}(\sqrt{\eta})\right)\right] \\
& +\left[-\frac{35 N^{3}+63 N^{2}+73 N+81}{27(N-1) N(N+1)^{2}} S_{2}+\frac{-5 N^{3}+23 N^{2}+33 N+41}{9(N-1) N(N+1)^{2}} H_{0}^{2}(\eta)\right. \\
& +\frac{2 P_{1}}{27(N-1)^{2} N^{2}(N+1)^{3}} H_{0}(\eta)-\frac{23 N^{3}+19 N^{2}+21 N+13}{9(N-1) N(N+1)^{2}} \zeta_{2} \\
& \left.+\frac{P_{12}}{810(N-1)^{3} N^{3}(N+1)^{4}(2 N-5)(2 N-3)(2 N-1) \eta}\right] S_{1} \\
& +\left[\frac{P_{5}}{81(N-1)^{2} N^{2}(N+1)^{3}}-\frac{13 N^{3}+21 N^{2}+23 N+27}{9(N-1) N(N+1)^{2}} H_{0}(\eta)\right] S_{1}^{2} \\
& +\left[\frac{P_{2}}{81(N-1)^{2} N^{2}(N+1)^{3}}+\frac{P_{7}}{45(N-1) N(N+1)^{2} \eta} H_{0}(\eta)\right] S_{2} \\
& -\frac{2 P_{8}}{45(N-1) N(N+1)^{2} \eta}\left[H_{0}^{2}(\eta)+2 S_{1,1}\left(\frac{\eta-1}{\eta}, 1, N\right)\right] S_{1}\left(\frac{\eta}{\eta-1}, N\right)
\end{aligned}
$$




$$
\begin{aligned}
& +\left[\frac{P_{13}}{810(N-1)^{3} N^{3}(N+1)^{4}(2 N-5)(2 N-3)(2 N-1) \eta}\right. \\
& \left.-\frac{13 N^{3}+21 N^{2}+23 N+27}{3(N-1) N(N+1)^{2}} \zeta_{2}\right] H_{0}(\eta)+\left[\frac{P_{10}}{540(N-1)^{2} N^{2}(N+1)^{3} \eta}\right. \\
& \left.\left.-\frac{P_{15}}{360(N-1) N(N+1) \eta^{3 / 2}} H_{-1}(\sqrt{\eta})\right] H_{0}^{2}(\eta)\right\}
\end{aligned}
$$

where the $P_{i}$ 's are polynomials in $N$ and $\eta$, and

$$
S_{b, \vec{a}}(y, \vec{x}, N)=\sum_{k=1}^{N} \frac{y^{k}}{k^{b}} S_{\vec{a}}(\vec{x}, k), \quad S_{\emptyset}=1, \quad b, a_{i} \in \mathbb{Z} \backslash\{0\}, \quad y, x_{i} \in \mathbb{C}
$$

are generalized harmonic sums [45]. The Mellin inversion of this result can be performed using the package HarmonicSums. The results are given in terms of generalized iterated integrals defined by

$$
G\left(\left\{f_{1}(\tau), f_{2}(\tau), \cdots, f_{n}(\tau)\right\}, z\right)=\int_{0}^{z} d \tau_{1} f_{1}\left(\tau_{1}\right) G\left(\left\{f_{2}(\tau), \cdots, f_{n}(\tau)\right\}, \tau_{1}\right)
$$

with

$$
G\left(\{\underbrace{\frac{1}{\tau}, \frac{1}{\tau}, \cdots, \frac{1}{\tau}}_{\mathrm{n} \text { times }}\}, z\right) \equiv \frac{1}{n !} \ln ^{n}(z) .
$$

These will also appear in the case of the pure singlet OME discussed in the next section. The letters in the alphabet of these iterated integrals, i.e., the functions $f_{i}(\tau)$ in Eq. (3.3), may in general depend on $\eta$.

\section{The pure singlet $\mathrm{OME}$}

In the case of the pure singlet two-mass contribution, the generic pole structure is given by

$$
\begin{aligned}
\hat{\hat{\tilde{A}}}_{Q q}^{(3), \mathrm{PS}}= & \frac{16}{3 \varepsilon^{3}} \gamma_{g q}^{(0)} \hat{\gamma}_{q g}^{(0)} \beta_{0, Q}+\frac{1}{\varepsilon^{2}}\left[4 \gamma_{g q}^{(0)} \hat{\gamma}_{q g}^{(0)} \beta_{0, Q}\left(L_{1}+L_{2}\right)+\frac{2}{3} \hat{\gamma}_{q g}^{(0)} \hat{\gamma}_{g q}^{(1)}-\frac{8}{3} \beta_{0, Q} \hat{\gamma}_{q q}^{\mathrm{PS},(1)}\right] \\
& +\frac{1}{\varepsilon}\left[2 \gamma_{g q}^{(0)} \hat{\gamma}_{q g}^{(0)} \beta_{0, Q}\left(L_{1}^{2}+L_{1} L_{2}+L_{2}^{2}\right)+\left\{\frac{1}{2} \hat{\gamma}_{q g}^{(0)} \hat{\gamma}_{g q}^{(1)}-2 \beta_{0, Q} \hat{\gamma}_{q q}^{\mathrm{PS},(1)}\right\}\left(L_{2}+L_{1}\right)\right. \\
& \left.+\frac{2}{3} \hat{\tilde{\gamma}}_{q q}^{(2), \mathrm{PS}}-8 a_{Q q}^{(2), \mathrm{PS}} \beta_{0, Q}+2 \hat{\gamma}_{q g}^{(0)} a_{g q}^{(2)}\right]+\tilde{a}_{Q q}^{(3), \mathrm{PS}} .
\end{aligned}
$$

In the $\overline{\mathrm{MS}}$-scheme, one obtains the following renormalized expression

$$
\begin{aligned}
\tilde{A}_{Q q}^{(3), \overline{\mathrm{MS}}, \mathrm{PS}}= & -\frac{1}{2} \gamma_{g q}^{(0)} \hat{\gamma}_{q g}^{(0)} \beta_{0, Q}\left(L_{2}^{2} L_{1}+L_{1}^{2} L_{2}+\frac{4}{3} L_{1}^{3}+\frac{4}{3} L_{2}^{3}\right)-\left\{\frac{1}{4} \hat{\gamma}_{q g}^{(0)} \hat{\gamma}_{g q}^{(1)}-\beta_{0, Q} \hat{\gamma}_{q q}^{\mathrm{PS},(1)}\right\}\left(L_{2}^{2}+L_{1}^{2}\right) \\
& +\left\{4 a_{Q q}^{(2), \mathrm{PS}} \beta_{0, Q}-\hat{\gamma}_{q g}^{(0)} a_{g q}^{(2)}-\frac{1}{2} \beta_{0, Q} \zeta_{2} \gamma_{g q}^{(0)} \hat{\gamma}_{q g}^{(0)}\right\}\left(L_{1}+L_{2}\right)+8 \bar{a}_{Q q}^{(2), \mathrm{PS}} \beta_{0, Q}-2 \hat{\gamma}_{q g}^{(0)} \bar{a}_{g q}^{(2)} \\
& +\tilde{a}_{Q q}^{(3), \mathrm{PS}} .
\end{aligned}
$$

There are sixteen diagrams contributing in this case, three of which are shown in Figure 5. All others are related to these by the exchange of masses and/or reversal of fermion lines. 


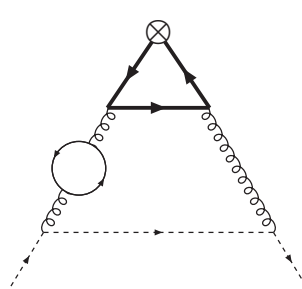

(1)

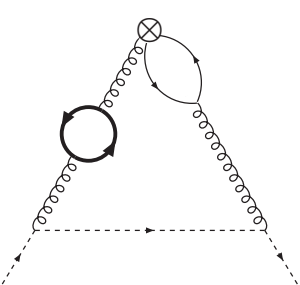

(2)

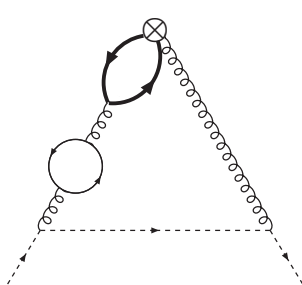

(3)

Figure 5: Diagrams for the two-mass contributions to $\tilde{A}_{Q q}^{(3), P S}$. The curly lines denote gluons, the dashed arrow line represents the external massless quarks, while the thick solid arrow line represents a quark of mass $m_{1}$, and the thin arrow line a quark of mass $m_{2}$. We assume $m_{1}>m_{2}$.

We use the same trick that we used before for $\tilde{A}_{q q}^{\mathrm{NS}}$ and $\tilde{A}_{g q}$ to decouple the mass coming from the fermion loop without the operator insertion. For the fermion loop with the vertex operator insertion, we use

$$
\underset{\mu, a}{\infty} \underset{\nu, b}{\infty}=16 \delta_{a b} T_{F} g_{s}^{2} \frac{(\Delta \cdot k)^{N-2}}{(4 \pi)^{D / 2}} \Gamma(2-D / 2) \int_{0}^{1} d x x^{N}(1-x) \frac{(\Delta \cdot k) \Delta_{\mu} k_{v}-k^{2} \Delta_{\mu} \Delta_{v}}{\left(m^{2}-x(1-x) k^{2}\right)^{2-D / 2}} \text {. }
$$

One obtains the following result for diagram 3 in Figure 5,

$$
\begin{aligned}
D_{3}(N)= & -64 C_{F} T_{F}^{2}\left(1+(-1)^{N}\right)\left(\frac{m_{2}^{2}}{\mu^{2}}\right)^{\frac{3}{2} \varepsilon}\left(1+\frac{\varepsilon}{2}\right) \frac{\Gamma(N-1)}{\Gamma(N+1+\varepsilon / 2)} \\
& \times \frac{1}{2 \pi i} \int_{-i \infty}^{+i \infty} d \sigma \eta^{-\sigma} \frac{\Gamma(-\sigma+N+1+\varepsilon / 2) \Gamma(-\sigma+2+\varepsilon / 2)}{\Gamma(-2 \sigma+N+3+\varepsilon)} \Gamma(-\sigma) \Gamma(-\sigma+\varepsilon) \\
& \times \Gamma\left(\sigma-\frac{3}{2} \varepsilon\right) \Gamma(\sigma-\varepsilon / 2) \frac{\Gamma^{2}(\sigma+2-\varepsilon)}{\Gamma(2 \sigma+4-2 \varepsilon)}
\end{aligned}
$$

The contour integral in Eq. (4.4) can be performed using the Mathematica package MB [52], together with the extension MBresolve [53], which allows to resolve the singularity structure in $\varepsilon$ of this type of integrals by taking residues in $\sigma$. Once this is done, we can expand in $\varepsilon$. At $O\left(\varepsilon^{0}\right)$, we are left with a sum of terms containing the poles in $\varepsilon$ of the integral and the original integral with $\varepsilon$ set to zero.

In the case where the operator insertion lies on a fermion line, we use the following expression,

$$
\begin{array}{rl}
\underset{\mu, a}{\infty} \underbrace{}_{\nu, b} & 4 \delta_{a b} T_{F} g_{s}^{2} \frac{(\Delta \cdot k)^{N-2}}{(4 \pi)^{D / 2}} \int_{0}^{1} d x x^{N-2}(1-x)[ \\
& -2\left(x(1-x)\left(g_{\mu v} k^{2}-2 k_{\mu} k_{v}\right)+m^{2} g_{\mu v}\right) \frac{x^{2} \Gamma(3-D / 2)(\Delta \cdot k)^{2}}{\left(m^{2}-x(1-x) k^{2}\right)^{3-D / 2}} \\
& +\Gamma(2-D / 2)(2 N x+1-N) \frac{x\left(k_{\mu} \Delta_{v}+k_{v} \Delta_{\mu}\right)(\Delta \cdot k)}{\left(m^{2}-x(1-x) k^{2}\right)^{2-D / 2}} \\
& +\Gamma(2-D / 2)((N-1)(1-2 x)-D x) \frac{x g_{\mu v}(\Delta \cdot k)^{2}}{\left(m^{2}-x(1-x) k^{2}\right)^{2-D / 2}}
\end{array}
$$




$$
\left.-\Gamma(1-D / 2) \frac{N-1}{1-x}(N(1-x)-1) \frac{\Delta_{\mu} \Delta_{v}}{\left(m^{2}-x(1-x) k^{2}\right)^{1-D / 2}}\right],
$$

which allows us to write the result for diagram 1 in Figure 5 (as well as all related diagrams) in terms of a linear combination of contour integrals similar to the one appearing in Eq. (4.4).

The $\Gamma$-functions in the integrand of Eq. (4.4) arise from the integration of Feynman parameters into Euler Beta-functions. In Ref. [18], we left one of these Feynman parameters unintegrated, since it was already in the form of a Mellin transform. In the case of Eq. (4.4) this refers to the term

$$
\frac{\Gamma(-\sigma+N+1+\varepsilon / 2) \Gamma(-\sigma+2+\varepsilon / 2)}{\Gamma(-2 \sigma+N+3+\varepsilon)}=\int_{0}^{1} d x x^{-\sigma+N+\varepsilon / 2}(1-x)^{-\sigma+1+\varepsilon / 2} .
$$

The exchange $m_{1} \leftrightarrow m_{2}$ implies also the change $\eta \rightarrow 1 / \eta$, which means that in Ref. [18], for some of the diagrams the integrals depended on $(\eta x(1-x))^{-1}$, while for other diagrams, the dependence was on $\frac{\eta}{x(1-x)}$. In the former case, we can close the contour to the left and take residues, since $(\eta x(1-x))^{-1}>4$ for all values of $x \in(0,1)$. The latter case is a bit more complicated, since $\frac{\eta}{x(1-x)}$ will be smaller or bigger than 4 , for $\eta<1$, depending on the value of $x$, which means that in some regions of $x$ we had to close the contours to the left, while in the remaining regions we had to close it to the right. On top of this, there are factors depending on $N$, such as the factor $\Gamma(N-1) / \Gamma(N+1+\varepsilon / 2)$ in (4.4), which after the $\varepsilon$ expansion, lead to factors of the form $1 / N$ and $1 /(N+1)$. These factors were absorbed into the Mellin transform using integration by parts, and the final result was then given in terms of integrals weighted by Heaviside $\theta$-functions.

In these proceedings we show an alternative method of calculating the pure singlet diagrams. We work directly in the representation of the diagrams with the fully integrated Feynman parameters. Let us consider the contour integral in (4.4). After the $\varepsilon$ expansion, one of the terms we get is given by

$$
J=\frac{1}{N+1} \frac{1}{2 \pi i} \int_{-1 / 2-i \infty}^{-1 / 2+i \infty} d \sigma \eta^{-\sigma} \frac{\Gamma(N+1-\sigma) \Gamma(-\sigma)^{2} \Gamma(\sigma)^{2} \Gamma(2-\sigma) \Gamma(2+\sigma)^{2}}{\Gamma(N+3-2 \sigma) \Gamma(4+2 \sigma)} .
$$

There is also a term with the same contour integral but a factor $1 / N$ instead of $1 /(N+1)$ in the decomposition of the diagram, and in the case of the diagrams where the operator insertion lies on a fermion line, also the factor $1 /(N-1)$ appears in some cases.

Closing the contour to the left and summing residues, we obtain the following result

$$
J=\eta \frac{\Gamma(N+1)}{\Gamma(N+4)}\left(4 S_{1}(N+3)-2 S_{1}(N)-2 \ln (\eta)-3\right)+J^{\prime}
$$

where

$$
\begin{aligned}
J^{\prime}= & \sum_{k=2}^{\infty} \frac{\eta^{k}}{N+1} B(k+N+1, k+2) \frac{\Gamma(k)^{2} \Gamma(2 k-3)}{\Gamma(k-1)^{2} \Gamma(k+1)^{2}}\left\{\ln ^{2}(\eta)+2 \ln (\eta)\left[S_{1}(k+N)\right.\right. \\
& \left.-2 S_{1}(2 k+N+2)-2 S_{1}(k-2)-\frac{2}{k}+S_{1}(k+1)+2 S_{1}(2 k-4)\right]+\frac{2}{k^{2}} \\
& +\left(S_{1}(k+N)-2 S_{1}(2 k+N+2)-2 S_{1}(k-2)-\frac{2}{k}+S_{1}(k+1)+2 S_{1}(2 k-4)\right)^{2}
\end{aligned}
$$




$$
\left.-S_{2}(k+N)+4 S_{2}(2 k+N+2)+2 S_{2}(k-2)-S_{2}(k+1)-4 S_{2}(2 k-4)\right\} .
$$

Unfortunately, this sum, as it stands, cannot be done using Sigma. Therefore, we proceed as follows. First we reintroduce an integral in $x$ using $^{3}$

$$
\begin{aligned}
\int_{0}^{1} d x x^{k+N}(1-x)^{k+1}= & B(k+N+1, k+2) \\
\int_{0}^{1} d x x^{k+N}(1-x)^{k+1} \ln (x)= & B(k+N+1, k+2)\left(S_{1}(k+N)-S_{1}(2 k+N+2)\right) \\
\int_{0}^{1} d x x^{k+N}(1-x)^{k+1} \ln (1-x)= & B(k+N+1, k+2)\left(S_{1}(k+1)-S_{1}(2 k+N+2)\right) \\
\int_{0}^{1} d x x^{k+N}(1-x)^{k+1} \ln (x)^{2}= & B(k+N+1, k+2)\left[\left(S_{1}(k+N)-S_{1}(2 k+N+2)\right)^{2}\right. \\
& \left.-S_{2}(k+N)+S_{2}(2 k+N+2)\right] \\
\int_{0}^{1} d x x^{k+N}(1-x)^{k+1} \ln (1-x)^{2}= & B(k+N+1, k+2)\left[\left(S_{1}(k+1)-S_{1}(2 k+N+2)\right)^{2}\right. \\
& \left.-S_{2}(k+1)+S_{2}(2 k+N+2)\right] \\
\int_{0}^{1} d x x^{k+N}(1-x)^{k+1} \ln (x) \ln (1-x)= & B(k+N+1, k+2)\left[-\zeta(2)+S_{2}(2 k+N+2)\right. \\
& +\left(S_{1}(k+1)-S_{1}(2 k+N+2)\right) \\
& \left.\times\left(S_{1}(k+N)-S_{1}(2 k+N+2)\right)\right]
\end{aligned}
$$

which leads to

$$
\begin{aligned}
J^{\prime}= & \sum_{k=2}^{\infty} \frac{\eta^{k}}{N+1} \int_{0}^{1} d x(1-x)^{k+1} x^{k+N} \frac{\Gamma(k)^{2} \Gamma(2 k-3)}{\Gamma(k-1)^{2} \Gamma(k+1)^{2}}\left\{\ln ^{2}(\eta)+2 \ln (\eta)\left[-2 S_{1}(k-2)\right.\right. \\
& \left.-\frac{2}{k}+2 S_{1}(2 k-4)+\ln (1-x)+\ln (x)\right]-4\left[S_{1}(k-2)+\frac{1}{k}-S_{1}(2 k-4)\right](\ln (1-x)+\ln (x)) \\
& +4\left(S_{1}(k-2)+\frac{1}{k}-S_{1}(2 k-4)\right)^{2}+2 \zeta_{2}+2 S_{2}(k-2)+\frac{2}{k^{2}}-4 S_{2}(2 k-4) \\
& \left.+(\ln (1-x)+\ln (x))^{2}\right\}
\end{aligned}
$$

Now we do the binomial expansion of the term $(1-x)^{k+1}$ and use

$$
\frac{1}{N+l} \int_{0}^{1} d x x^{N} f(x)=\int_{0}^{1} d x x^{N+l-1}\left(\int_{0}^{1} d y y^{-l} f(y)-\int_{0}^{x} d y y^{-l} f(y)\right)
$$

in order to absorb the $\frac{1}{N+l}$ factor into the integrand. The integrals from 0 to $x$ can be done using

$$
\int_{0}^{x} d y y^{n} \ln (y)=\frac{x^{n+1}}{n+1} \ln (x)-\frac{x^{n+1}}{(n+1)^{2}}
$$

\footnotetext{
${ }^{3}$ Transformations of this kind have been used in Ref. [54] before.
} 


$$
\begin{aligned}
\int_{0}^{x} d y y^{n} \ln (1-y)= & -\frac{S_{1}(x, n)}{n+1}-\frac{x^{n+1}}{(n+1)^{2}}+\frac{x^{n+1}-1}{n+1} \ln (1-x) \\
\int_{0}^{x} d y y^{n} \ln (y)^{2}= & \frac{2 x^{n+1}}{(n+1)^{3}}+\frac{x^{n+1}}{n+1} \ln ^{2}(x)-\frac{2 x^{n+1}}{(n+1)^{2}} \ln (x) \\
\int_{0}^{x} d y y^{n} \ln (1-y)^{2}= & \frac{\ln ^{2}(x)}{n+1}\left[\frac{2}{n+1} S_{1}(x, n+1)+2 S_{1,1}(\{1, x\}, n)-2 x\right. \\
& +2 \ln (1-x)\left(S_{1}(n+1)-S_{1}(x, n+1)-1+x\right)+2 x \\
\int_{0}^{x} d y y^{n} \ln (y) \ln (1-y)= & \frac{1}{(n+1)^{2}}\left[S_{1}(x, n-1)-x^{n+1} \ln (x)-\left(x^{n+1}-1\right) \ln (1-x)\right] \\
& -\frac{x^{n} \ln (x)}{n(n+1)}+\frac{1}{n+1}\left[S_{2}(x, n-1)-\ln (x) S_{1}(x, n-1)-\operatorname{Li}_{2}(x)\right. \\
& \left.+\left(x^{n+1}-1\right) \ln (x) \ln (1-x)\right]+\frac{(2 n+1) x^{n}}{n^{2}(n+1)^{2}}+\frac{2 x^{n+1}}{(n+1)^{3}} .
\end{aligned}
$$

From this we get,

$$
\begin{aligned}
& J^{\prime}=\int_{0}^{1} d x x^{N+1} \sum_{k=2}^{\infty} \sum_{i=0}^{k+1}(-1)^{i} \eta^{k}\left(\begin{array}{c}
k+1 \\
i
\end{array}\right) \frac{\Gamma(k)^{2} \Gamma(2 k-3)}{\Gamma(k-1)^{2} \Gamma(k+1)^{2}}\left\{\frac{8\left(1-x^{i+k-1}\right)}{(i+k-1)^{3}}\right. \\
& +\frac{1}{(i+k-1)^{2}}\left[-4 S_{1}(x, i+k-2)+4 S_{1}(i+k-2)+\left(1-x^{i+k-1}\right)\left(8 S_{1}(k-2)\right.\right. \\
& \left.\left.+\frac{8}{k}-8 S_{1}(2 k-4)-4 \ln (\eta)\right)+4 x^{i+k-1} \ln (x)+2\left(2 x^{i+k-1}-1\right) \ln (1-x)\right] \\
& +\frac{1}{i+k-1}\left\{\ln (\eta)\left(2 S_{1}(x, i+k-2)-2 S_{1}(i+k-2)-2 x^{i+k-1} \ln (x)\right)\right. \\
& +\ln (x)\left(2 S_{1}(x, i+k-2)+4 x^{i+k-1} S_{1}(k-2)+\frac{4}{k} x^{i+k-1}-4 x^{i+k-1} S_{1}(2 k-4)\right) \\
& +4\left(S_{1}(k-2)+\frac{1}{k}-S_{1}(2 k-4)\right)\left(S_{1}(i+k-2)-S_{1}(x, i+k-2)\right)+2 \operatorname{Li}_{2}(x) \\
& -2 S_{2}(x, i+k-2)-2 S_{1,1}(\{1, x\}, i+k-2)-\frac{\pi^{2}}{3} x^{i+k-1}-x^{i+k-1} \ln ^{2}(x) \\
& +\ln (1-x)\left(2 S_{1}(x, i+k-2)-2 S_{1}(i+k-1)\right)+2 S_{2}(i+k-2)+2 S_{1,1}(i+k-2) \\
& +\left(1-x^{i+k-1}\right)\left[\ln (\eta)\left(-4 S_{1}(k-2)-\frac{4}{k}+4 S_{1}(2 k-4)+2 \ln (1-x)\right)\right. \\
& -4 \ln (1-x)\left(S_{1}(k-2)+\frac{1}{k}-S_{1}(2 k-4)\right)+4\left(S_{1}(k-2)+\frac{1}{k}-S_{1}(2 k-4)\right)^{2} \\
& \left.\left.\left.+2 S_{2}(k-2)+\frac{2}{k^{2}}-4 S_{2}(2 k-4)+\ln ^{2}(\eta)+\ln ^{2}(1-x)+2 \ln (x) \ln (1-x)\right]\right\}\right\} \text {. }
\end{aligned}
$$

Now we can do perform the double sum using Sigma and Harmoni cSums. We finally obtain, $J^{\prime}=\int_{0}^{1} d x x^{N+1}\left\{-\frac{1}{24} G^{2}\left(\left\{\frac{1}{\tau}, \frac{\sqrt{4 \tau-1}}{\tau}\right\}, x \eta-x^{2} \eta\right)-\frac{1}{12} i G\left(\left\{\frac{\sqrt{4 \tau-1}}{\tau}, \frac{1}{\tau}, \frac{1}{\tau}, \frac{1}{\tau}\right\}, x-x^{2}\right)\right.$ 


$$
\begin{aligned}
& +\frac{1}{288} \ln ^{4}((1-x) x \eta)+\frac{12 x^{2}-x+6}{216 x} \ln ^{3}((1-x) x \eta)+\left[\frac{\zeta_{2}}{24}+\frac{66 x^{2}-15 \eta x-37 x+24}{108 x}\right. \\
& \left.-\frac{11}{36} \ln (1-\eta)\right] \ln ^{2}((1-x) x \eta)+\left[\frac{T_{2}}{54 x}+\ln (1-x)\left(\frac{5 \eta}{36}+\frac{11}{36} \ln (1-\eta)\right)\right. \\
& \left.+\ln (x)\left(\frac{11}{12} \ln (1-\eta)-\frac{7 \eta}{12}\right)-\frac{11}{9} x \ln (1-\eta)\right] \ln ((1-x) x \eta)+\frac{\zeta_{2} T_{3}}{27 x}+\frac{\eta T_{1}}{810}-\zeta_{2} \eta \\
& +\left\{\frac{11}{36} G\left(\left\{\frac{\sqrt{4 \tau-1} \sqrt{4 \eta \tau-1}}{\tau}\right\}, x-x^{2}\right)-\frac{20}{9} \eta G\left(\{\sqrt{4 \tau-1} \sqrt{4 \eta \tau-1}\}, x-x^{2}\right)\right\}\left[\zeta_{2}\right. \\
& \left.+i G\left(\left\{\frac{1}{\tau}, \frac{\sqrt{4 \tau-1}}{\tau}\right\}, x \eta-x^{2} \eta\right)\right]+\frac{20}{9} i \eta G\left(\left\{\frac{1}{\tau}, \frac{\sqrt{4 \eta \tau-1}}{\tau}, \sqrt{4 \tau-1} \sqrt{4 \eta \tau-1}\right\}, x-x^{2}\right) \\
& +\frac{1}{12} i G\left(\left\{\frac{1}{\tau}, \frac{\sqrt{4 \tau-1}}{\tau}\right\}, x \eta-x^{2} \eta\right)\left[\zeta_{2}+G\left(\left\{\frac{\sqrt{4 \tau-1}}{\tau}, \frac{\sqrt{4 \eta \tau-1}}{\tau}\right\}, x-x^{2}\right)\right] \\
& -\frac{11}{36} i G\left(\left\{\frac{1}{\tau}, \frac{\sqrt{4 \eta \tau-1}}{\tau}, \frac{\sqrt{4 \tau-1} \sqrt{4 \eta \tau-1}}{\tau}\right\}, x-x^{2}\right)+\frac{T_{3}}{27 x}(1-4 x(1-x) \eta)^{3 / 2}\left[-\zeta_{2}\right. \\
& \left.+2 i\left(1+\frac{1}{2} \ln ((1-x) x \eta)\right) G\left(\left\{\frac{\sqrt{4 \tau-1}}{\tau}\right\}, x \eta-x^{2} \eta\right)-i G\left(\left\{\frac{1}{\tau}, \frac{\sqrt{4 \tau-1}}{\tau}\right\}, x \eta-x^{2} \eta\right)\right] \\
& +\frac{1}{12} i G\left(\left\{\frac{1}{\tau}, \frac{\sqrt{4 \eta \tau-1}}{\tau}, \frac{\sqrt{4 \eta \tau-1}}{\tau}, \frac{\sqrt{4 \tau-1}}{\tau}\right\}, x-x^{2}\right)+\ln ^{2}(x)\left(\frac{7 \eta}{24}-\frac{11}{24} \ln (1-\eta)\right) \\
& +\ln ^{2}(1-x)\left(-\frac{5 \eta}{72}-\frac{11}{72} \ln (1-\eta)\right)+\ln (1-x)\left(\frac{11}{18} \ln (1-\eta)-\frac{1}{27} \eta(8 \eta-29)\right) \\
& +\ln (x)\left[-\frac{1}{54} \eta(16 \eta-169)+\ln (1-x)\left(\frac{7 \eta}{12}-\frac{11}{12} \ln (1-\eta)\right)+\frac{11}{18} \ln (1-\eta)\right] \\
& -\frac{i}{12}\left(\ln (\eta)+\frac{17}{3}\right) G\left(\left\{\frac{\sqrt{4 \tau-1}}{\tau}, \frac{1}{\tau}, \frac{1}{\tau}\right\}, x-x^{2}\right)+\left(\frac{\ln (x)}{6}-\frac{\ln (\eta)}{3}\right) \operatorname{Li}_{3}(\eta)+\frac{\operatorname{Li}_{4}(\eta)}{2} \\
& +\left(\frac{1}{12} \ln ((1-x) x \eta)-\frac{12 x^{2}-13 x+6}{36 x}\right) G\left(\left\{\frac{1}{\tau}, \frac{\sqrt{4 \tau-1}}{\tau}, \frac{\sqrt{4 \tau-1}}{\tau}\right\}, x \eta-x^{2} \eta\right) \\
& +\left(1+\frac{1}{2} \ln ((1-x) x \eta)\right)\left\{-\frac{1}{6} i G\left(\left\{\frac{\sqrt{4 \tau-1}}{\tau}, \frac{\sqrt{4 \eta \tau-1}}{\tau}, \frac{\sqrt{4 \eta \tau-1}}{\tau}\right\}, x-x^{2}\right)\right. \\
& +\frac{T_{4}}{36 x} G^{2}\left(\left\{\frac{\sqrt{4 \tau-1}}{\tau}\right\}, x \eta-x^{2} \eta\right)+\left[\frac{40}{9} i \eta G\left(\{\sqrt{4 \tau-1} \sqrt{4 \eta \tau-1}\}, x-x^{2}\right)\right. \\
& \left.\left.-\frac{11}{18} i G\left(\left\{\frac{\sqrt{4 \tau-1} \sqrt{4 \eta \tau-1}}{\tau}\right\}, x-x^{2}\right)\right] G\left(\left\{\frac{\sqrt{4 \tau-1}}{\tau}\right\}, x \eta-x^{2} \eta\right)\right\} \\
& -i \eta\left(\frac{40}{9}+\frac{20}{9} \ln ((1-x) x \eta)\right) G\left(\left\{\frac{\sqrt{4 \eta \tau-1}}{\tau}, \sqrt{4 \tau-1} \sqrt{4 \eta \tau-1}\right\}, x-x^{2}\right) \\
& +i\left(\frac{11}{18}+\frac{11}{36} \ln ((1-x) x \eta)\right) G\left(\left\{\frac{\sqrt{4 \eta \tau-1}}{\tau}, \frac{\sqrt{4 \tau-1} \sqrt{4 \eta \tau-1}}{\tau}\right\}, x-x^{2}\right) \\
& +\frac{T_{4}}{36 x}\left[i \zeta_{2} G\left(\left\{\frac{\sqrt{4 \tau-1}}{\tau}\right\}, x \eta-x^{2} \eta\right)-G\left(\left\{\frac{\sqrt{4 \tau-1}}{\tau}, \frac{1}{\tau}, \frac{\sqrt{4 \tau-1}}{\tau}\right\}, x \eta-x^{2} \eta\right)\right. \\
& \left.+\zeta_{2} \ln ((1-x) x \eta)\right]+\operatorname{Li}_{2}(x)\left(\frac{13 \eta}{18}-\frac{11}{18} \ln (1-\eta)+\frac{\mathrm{Li}_{2}(\eta)}{6}\right)-\frac{1}{6} \ln (1-x) \operatorname{Li}_{3}(\eta)
\end{aligned}
$$




$$
\begin{aligned}
& +G\left(\left\{\frac{\sqrt{4 \tau-1}}{\tau}, \frac{\sqrt{4 \eta \tau-1}}{\tau}\right\}, x-x^{2}\right)\left(\frac{\zeta_{2}}{12}+\frac{\ln (\eta)}{6}+\frac{1}{12} \ln (\eta) \ln ((1-x) x \eta)\right) \\
& +\frac{i}{12} G\left(\left\{\frac{1}{\tau}, \frac{\sqrt{4 \tau-1}}{\tau}\right\}, x-x^{2}\right)\left[\frac{1}{2} \ln ^{2}(\eta)+\frac{17}{3} \ln (\eta)+\zeta_{2}-\frac{1}{3}(5 \eta-22)-\frac{11}{3} \ln (1-\eta)\right. \\
& \left.+\mathrm{Li}_{2}(\eta)\right]+\left[\frac{1}{24} \ln ^{2}(1-x)+\left(\frac{1}{36}(12 x-17)+\frac{1}{12} \ln (x \eta)\right) \ln (1-x)+\frac{\ln ^{2}(\eta)}{8}-\frac{1}{24} \ln ^{2}(x \eta)\right. \\
& \left.+\frac{1}{18} x(1+\sqrt{\eta})(5 \eta-5 \sqrt{\eta}-22)+\frac{1}{36}(12 x+5) \ln (x)+\frac{1}{18}(6 x-11) \ln (\eta)\right] \operatorname{Li}_{2}(\eta) \\
& +\sqrt{\eta}(5 \eta-27)\left\{\operatorname { l n } ( \frac { 1 + \sqrt { \eta } } { 1 - \sqrt { \eta } } ) \left[\frac{1}{144} \ln ^{2}(1-x)+\left(\frac{1}{36}(2 x-1)+\frac{1}{72} \ln (x \eta)\right) \ln (1-x)\right.\right. \\
& +\frac{\ln ^{2}(\eta)}{48}-\frac{1}{144} \ln ^{2}(x \eta)+\frac{1}{72} i G\left(\left\{\frac{1}{\tau}, \frac{\sqrt{4 \tau-1}}{\tau}\right\}, x-x^{2}\right)+\frac{1}{36}(2 x-1) \ln (x)+\frac{x}{18} \ln (\eta) \\
& \left.+\frac{\mathrm{Li}_{2}(x)}{36}\right]+\left(-\frac{2 x}{9}-\frac{1}{18} \ln (1-x)+\frac{\ln (x)}{18}-\frac{\ln (\eta)}{9}\right) \operatorname{Li}_{2}(\sqrt{\eta})+\frac{2}{9} \operatorname{Li}_{3}(\sqrt{\eta})-\frac{\mathrm{Li}_{3}(\eta)}{36} \\
& -\frac{i}{18} G\left(\left\{\frac{\sqrt{4 \tau-1}}{\tau}\right\}, x-x^{2}\right)[ \\
& \frac{1}{2} \ln \left(\frac{1+\sqrt{\eta}}{1-\sqrt{\eta}}\right)\left(1+\frac{1}{2} \ln ((1-x) x \eta)\right)-\operatorname{Li}_{2}(\sqrt{\eta}) \\
& \left.\left.+\frac{\operatorname{Li}_{2}(\eta)}{4}\right]+\left(\frac{1}{72} \ln (1-x)-\frac{\ln (x)}{72}+\frac{\ln (\eta)}{36}\right) \operatorname{Li}_{2}(\eta)\right\}+\left[\frac{1}{36} i \ln ^{3}(\eta)-\frac{11}{72} i \ln ^{2}(\eta)\right. \\
& +\frac{1}{36} i(5 \eta-22) \ln (\eta)-\frac{1}{12} G\left(\left\{\frac{1}{\tau}, \frac{\sqrt{4 \tau-1}}{\tau}\right\}, x \eta-x^{2} \eta\right) \ln (\eta)-\frac{11 i \zeta_{2}}{36}-\frac{1}{27} i \eta(8 \eta-29) \\
& -\frac{1}{12} i G\left(\left\{\frac{1}{\tau}, \frac{\sqrt{4 \tau-1}}{\tau}, \frac{\sqrt{4 \tau-1}}{\tau}\right\}, x \eta-x^{2} \eta\right)+\frac{i}{36}\left(5 \eta-3 \zeta_{2}-22+11 \ln (1-\eta)\right. \\
& -17 \ln (\eta)) \ln ((1-x) x)+\frac{11}{18} i \ln (1-\eta)\left(1+\frac{\ln (\eta)}{2}\right)+\left(\frac{5 i}{36}-\frac{1}{12} i \ln ((1-x) x \eta)\right) \operatorname{Li}_{2}(\eta) \\
& \left.\left.+\frac{i \mathrm{Li}_{3}(\eta)}{6}\right] G\left(\left\{\frac{\sqrt{4 \tau-1}}{\tau}\right\}, x-x^{2}\right)+\frac{1}{18}(11-12 x) \operatorname{Li}_{3}(\eta)\right\},
\end{aligned}
$$

where

$$
\begin{aligned}
T_{1}= & 5\left(640 x^{3}-1011 x^{2}-171 x+810\right)+32 \eta x\left(144 x^{4}-360 x^{3}+320 x^{2}-120 x+15\right) \\
T_{2}= & 192 \eta^{2} x^{6}-512 \eta^{2} x^{5}+32 \eta(18 \eta+5) x^{4}-\eta(416 \eta+251) x^{3} \\
& +2\left(96 \eta^{2}+103 \eta+30\right) x^{2}-(231 \eta+32) x+12 \\
T_{3}= & 15 x^{2}-8 x+3 \\
T_{4}= & 12 x^{2}-7 x+6
\end{aligned}
$$

All other integrals appearing in the calculation can be done in a similar way. This way of calculating the diagrams applies perfectly well in all of the cases where the operator insertion lies on the heaviest of the quarks, as long as we are assuming that $\eta<1$. Taking into account that the diagrams where the insertion lies on the lightest quark are related to the former diagrams by the change $\eta \rightarrow 1 / \eta$, we can in principle obtain the results of the latter diagrams by analytic continuation to 
the region where $\eta>1$. This is, however, far less trivial compared with the $\tilde{A}_{g g, Q}$ case, since the square root letters in the alphabet of the iterated integrals introduce branch cuts that need to be analyzed carefully. The complete result given in Ref. [18] has been obtained in a different way.

\section{Conclusions}

We have presented the two-mass contributions for a series of three-loop OMEs. The simplest ones are $\tilde{A}_{q q, Q}^{(3), N S}$ and $\tilde{A}_{g q, Q}^{(3)}$, for which the $N$ and $\eta$ dependence factorizes. In the case of $\tilde{A}_{g g, Q}^{(3)}$, we presented results in the physical case, going beyond those given in [17], where a series of scalar diagrams were computed. In the pure singlet case, which was studied in [18], we presented a new way of computing the diagrams. This new way has the advantage of producing a result that is given entirely by iterated integrals, without the need to introduce additional integrations on top of these, as we did in [18]. However, the results so far are only valid for half of the diagrams. In order to cover the other half, the analytic continuation $\eta \rightarrow 1 / \eta$ still remain to be done.

With the full results for $\tilde{A}_{g g, Q}^{(3)}$ within grasp, and having calculated all the other OMEs presented here, the only remaining OME is $\tilde{A}_{Q g}^{(3)}$. Considering that even in the single mass case this OME exhibits integrals with an elliptic behaviour [55,56], it doesn't seem feasible that this OME will be computed analytically any time soon. New methods will need to be applied in this case, which we plan to study at some point in the future.

\section{References}

[1] S. Bethke et al., Workshop on Precision Measurements of $\alpha_{s}$, arXiv:1110.0016 [hep-ph];

S. Moch, S. Weinzierl et al., High precision fundamental constants at the TeV scale, arXiv:1405.4781 [hep-ph];

S. Alekhin, J. Blümlein and S.O. Moch, Mod. Phys. Lett. A 31 (2016) no.25, 1630023.

[2] A. Accardi et al., Eur. Phys. J. C 76 (2016) no.8, 471 [arXiv:1603.08906 [hep-ph]];

S. Alekhin, J. Blümlein, S. Moch and R. Placakyte, Phys. Rev. D 96 (2017) no.1, 014011 [arXiv:1701.05838 [hep-ph]].

[3] S. Alekhin, J. Blümlein, K. Daum, K. Lipka and S. Moch, Phys. Lett. B 720 (2013) 172 [arXiv:1212.2355 [hep-ph]];

A. Gizhko et al., Phys. Lett. B 775 (2017) 233 [arXiv:1705.08863 [hep-ph]].

[4] J. Ablinger, J. Blümlein, A. De Freitas, A. Hasselhuhn, A. von Manteuffel, M. Round, C. Schneider and F. Wißbrock, Nucl. Phys. B 882 (2014) 263 [arXiv:1402.0359 [hep-ph]].

[5] J. Ablinger, J. Blümlein, A. De Freitas, A. Hasselhuhn, A. von Manteuffel, M. Round and C. Schneider, Nucl. Phys. B 885 (2014) 280 [arXiv:1405.4259 [hep-ph]].

[6] J. Ablinger, A. Behring, J. Blümlein, A. De Freitas, A. Hasselhuhn, A. von Manteuffel, M. Round, C. Schneider, F. Wißbrock, Nucl. Phys. B 886 (2014) 733 [arXiv:1406.4654 [hep-ph]].

[7] A. Behring, J. Blümlein, A. De Freitas, A. von Manteuffel and C. Schneider, Nucl. Phys. B 897 (2015) 612 [arXiv:1504.08217 [hep-ph]].

[8] A. Behring, J. Blümlein, A. De Freitas, A. Hasselhuhn, A. von Manteuffel and C. Schneider, Phys. Rev. D 92 (2015) no.11, 114005 [arXiv:1508.01449 [hep-ph]]. 
[9] A. Behring, J. Blümlein, G. Falcioni, A. De Freitas, A. von Manteuffel and C. Schneider, Phys. Rev. D 94 (2016) no.11, 114006 [arXiv:1609.06255 [hep-ph]].

[10] J. Ablinger, A. Behring, J. Blümlein, A. De Freitas, A. von Manteuffel and C. Schneider, Nucl. Phys. B 890 (2014) 48 [arXiv:1409.1135 [hep-ph]].

[11] J. Ablinger, A. Behring, J. Blümlein, A. De Freitas, A. von Manteuffel and C. Schneider, Comput. Phys. Commun. 202 (2016) 33 [arXiv:1509.08324 [hep-ph]].

[12] J. Ablinger, A. Behring, J. Blümlein, A. De Freitas, A. von Manteuffel and C. Schneider, PoS (QCDEV2017) 031 [arXiv:1711.07957 [hep-ph]].

[13] J. Blümlein and C. Schneider, Phys. Lett. B 771 (2017) 31 [arXiv:1701.04614 [hep-ph]].

[14] A. Behring, I. Bierenbaum, J. Blümlein, A. De Freitas, S. Klein and F. Wißbrock, Eur. Phys. J. C 74 (2014) no.9, 3033 [arXiv:1403.6356 [hep-ph]].

[15] I. Bierenbaum, J. Blümlein and S. Klein, Nucl. Phys. B 820 (2009) 417 [arXiv:0904.3563 [hep-ph]].

[16] J. Blümlein, A. De Freitas, C. Schneider, and K. Schönwald, DESY 17-187.

[17] J. Ablinger, J. Blümlein, A. De Freitas, A. Hasselhuhn, C. Schneider and F. Wißbrock, Nucl. Phys. B 921 (2017) 585 [arXiv:1705.07030 [hep-ph]].

[18] J. Ablinger, J. Blümlein, A. De Freitas, C. Schneider and K. Schönwald, The two-mass contribution to the three-loop pure singlet operator matrix element, arXiv:1711.06717 [hep-ph].

[19] E.W. Barnes, Proc. Lond. Math. Soc. (2) 6 (1908) 141.

[20] E.W. Barnes, Quarterly Journal of Mathematics 41 (1910) 136.

[21] H. Mellin, Math. Ann. 68, no. 3 (1910) 305.

[22] E.T. Whittaker and G.N. Watson, A Course of Modern Analysis, (Cambridge University Press, Cambridge, 1927; reprinted 1996) 616 p.

[23] E.C. Titchmarsh, Introduction to the Theory of Fourier Integrals, (Calendron Press, Oxford, 1937; 2nd Edition 1948).

[24] J. Ablinger, J. Blümlein, S. Klein, C. Schneider and F. Wißbrock, Nucl. Phys. B 844 (2011) 26 [arXiv:1008.3347 [hep-ph]].

[25] J. Blümlein, A. Hasselhuhn, S. Klein and C. Schneider, Nucl. Phys. B 866 (2013) 196 [arXiv:1205.4184 [hep-ph]].

[26] W.N. Bailey, Generalized Hypergeometric Series, (Cambridge University Press, Cambridge, 1935); L.J. Slater, Generalized Hypergeometric Functions, (Cambridge University Press, Cambridge, 1966); P. Appell and J. Kampé de Fériet, Fonctions Hypergéométriques et Hyperspériques, Polynomes D' Hermite, (Gauthier-Villars, Paris, 1926);

P. Appell, Les Fonctions Hypergëométriques de Plusieur Variables, (Gauthier-Villars, Paris, 1925);

J. Kampé de Fériet, La fonction hypergëométrique,(Gauthier-Villars, Paris, 1937);

H. Exton, Multiple Hypergeometric Functions and Applications, (Ellis Horwood, Chichester, 1976);

H. Exton, Handbook of Hypergeometric Integrals, (Ellis Horwood, Chichester, 1978);

H.M. Srivastava and P.W. Karlsson, Multiple Gaussian Hypergeometric Series, (Ellis Horwood, Chicester, 1985);

M.J. Schlosser, in: Computer Algebra in Quantum Field Theory: Integration, Summation and Special Functions, C. Schneider, J. Blümlein, Eds., p. 305, (Springer, Wien, 2013) [arXiv:1305.1966 [math.CA]]. 
[27] M. Karr, J. ACM 28 (1981) 305.

[28] C. Schneider, Symbolic Summation in Difference Fields Ph.D. Thesis RISC, Johannes Kepler University, Linz technical report 01-17 (2001).

[29] C. Schneider, An. Univ. Timisoara Ser. Mat.-Inform. 42 (2004) 163;

J. Differ. Equations Appl. 11 (2005) 799;

Appl. Algebra Engrg. Comm. Comput. 16 (2005) 1.

[30] C. Schneider, J. Algebra Appl. 6 (2007) 415.

[31] C. Schneider, Motives, Quantum Field Theory, and Pseudodifferential Operators (Clay Mathematics Proceedings Vol. 12 ed. A. Carey, D. Ellwood, S. Paycha and S. Rosenberg,(Amer. Math. Soc) (2010), 285 [arXiv:0904.2323].

[32] C. Schneider, Ann. Comb. 14 (2010) 533 [arXiv:0808.2596].

[33] C. Schneider, in: Computer Algebra and Polynomials, Applications of Algebra and Number Theory, J. Gutierrez, J. Schicho, M. Weimann (ed.), Lecture Notes in Computer Science (LNCS) 8942 (2015), 157[arXiv:13077887 [cs.SC]].

[34] C. Schneider, J. Symbolic Comput. 43 (2008) 611 [arXiv:0808.2543v1]; J. Symb. Comput. 72 (2016) 82 [arXiv:1408.2776 [cs.SC]]; J. Symb. Comput. 80 (2017), 616 [arXiv:1603.04285 [cs.SC]].

[35] C. Schneider, Ann. Comb. 9(1) (2005) 75;

S.A. Abramov and M. Petkovšek, J. Symbolic Comput., 45(6) (2010) 684;

C. Schneider, Appl. Algebra Engrg. Comm. Comput., 21(1) (2010) 1;

C. Schneider, In: Symbolic and Numeric Algorithms for Scientific Computing (SYNASC), 2014, 15th International Symposium, F. Winkler, V. Negru, T. Ida, T. Jebelean, D. Petcu, S. Watt, D. Zaharie (ed.), (2015) pp. 26; IEEE Computer Society, arXiv:1412.2782v1 [cs.SC].

[36] C. Schneider, Sém. Lothar. Combin. 56 (2007) 1, article B56b.

[37] C. Schneider, Computer Algebra in Quantum Field Theory: Integration, Summation and Special Functions Texts and Monographs in Symbolic Computation eds. C. Schneider and J. Blümlein (Springer, Wien, 2013) 325, arXiv:1304.4134 [cs.SC].

[38] T. Huber and D. Maitre, Comput. Phys. Commun. 178 (2008) 755 [arXiv:0708.2443 [hep-ph]].

[39] L. Lewin, Dilogarithms and associated functions, (Macdonald, London, 1958).

[40] L. Lewin, Polylogarithms and associated functions, (North Holland, New York, 1981).

[41] A. Devoto and D.W. Duke, Riv. Nuovo Cim. 7N6 (1984) 1.

[42] J. Ablinger and J. Blümlein, Harmonic Sums, Polylogarithms, Special Numbers, and their Generalizations, arXiv:1304.7071 [math-ph], in : Integration, Summation and Special Functions in Quantum Field Theory, eds. J. Blümlein and C. Schneider, (Springer, Wien, 2013) 1.

[43] J. Ablinger, PoS (LL2014) 019; Computer Algebra Algorithms for Special Functions in Particle Physics, Ph.D. Thesis, J. Kepler University Linz, 2012, arXiv:1305.0687 [math-ph]; A Computer Algebra Toolbox for Harmonic Sums Related to Particle Physics, Diploma Thesis, J. Kepler University Linz, 2009, arXiv:1011.1176 [math-ph].

[44] J. Ablinger, J. Blümlein and C. Schneider, J. Math. Phys. 52 (2011) 102301 [arXiv:1105.6063 [math-ph]].

[45] J. Ablinger, J. Blümlein and C. Schneider, J. Math. Phys. 54 (2013) 082301 [arXiv:1302.0378 [math-ph]]. 
[46] J. Ablinger, J. Blümlein, S. Klein and C. Schneider, Nucl. Phys. Proc. Suppl. 205-206 (2010) 110 [arXiv:1006.4797 [math-ph]];

J. Blümlein, A. Hasselhuhn and C. Schneider, PoS (RADCOR 2011) 032 [arXiv:1202.4303 [math-ph]];

C. Schneider, J. Phys. Conf. Ser. 523 (2014) 012037 [arXiv:1310.0160 [cs.SC]].

[47] J.A.M. Vermaseren, Comput. Phys. Commun. 83 (1994) 45.

[48] J.A.M. Vermaseren, Int. J. Mod. Phys. A 14 (1999) 2037 [hep-ph/9806280]. J. Blümlein and S. Kurth, Phys. Rev. D 60 (1999) 014018 [arXiv:hep-ph/9810241].

[49] J. Ablinger, J. Blümlein, S. Klein, C. Schneider and F. Wißbrock, arXiv:1106.5937 [hep-ph].

[50] J. Ablinger, J. Blümlein, A. Hasselhuhn, S. Klein, C. Schneider and F. Wißbrock, PoS (RADCOR2011) 031 [arXiv:1202.2700 [hep-ph]].

[51] J. Blümlein, S. Klein and B. Tödtli, Phys. Rev. D 80 (2009) 094010 [arXiv:0909.1547 [hep-ph]].

[52] M. Czakon, Comput. Phys. Commun. 175 (2006) 559 [hep-ph/0511200];

[53] A.V. Smirnov and V.A. Smirnov, Eur. Phys. J. C 62 (2009) 445 [arXiv:0901.0386 [hep-ph]].

[54] I. Bierenbaum, J. Blümlein and S. Klein, Phys. Lett. B 648 (2007) 195 [hep-ph/0702265].

[55] J. Ablinger, J. Blümlein, A. De Freitas, M. van Hoeij, E. Imamoglu, C. G. Raab, C.-S. Radu and C. Schneider, arXiv:1706.01299 [hep-th].

[56] J. Ablinger et al., PoS(RADCOR2017)069 arXiv:1711.09742 [hep-ph]. 